\title{
Antioxidant and Anti-hyperglycemic Activity of Polysaccharide Isolated from Dendrobium chrysotoxum Lindl
}

\author{
Yaping Zhao ${ }^{1, \#}$, Young-Ok Son ${ }^{2,3}$, So-Soon Kim ${ }^{2,3}$, Yong-Suk Jang ${ }^{1,2}$ and Jeong-Chae Lee ${ }^{1,3, *}$ \\ ${ }^{1}$ Research Center of Bioactive Materials, ${ }^{2}$ Division of Biological Sciences, ${ }^{3}$ Laboratory of Cell Biology in Department of Orthodontics \\ and Institute of Oral Biosciences, Chonbuk National University, Chonju 561-756, Korea
}

Received 6 February 2007, Accepted 9 April 2007

\begin{abstract}
Although polysaccharide is believed to play an important role in the medicinal effect of Dendrobium chrysotoxum Lindl (DCL), its role as an antioxidant and in antihyperglycemic induction was not reported. In this study, polysaccharide with molecular weight of approximately $150 \mathrm{kDa}$, herein named DCLP, was isolated from the stem of DCL, and its antioxidative, hypoglycemic and immune stimulating effects were evaluated using various in vitro and in vivo assay systems. DCLP inhibited hydroxyl radicals ('OH)-mediated deoxyribose degradation by scavenging hydroxyl radicals directly as well as by chelating iron ions. DCLP also showed dose-dependent scavenging activity on superoxide anions $\left(\mathrm{O}_{2}{ }^{-}\right)$and offered significant protection $(p<0.001)$ against glucose oxidasemediated cytotoxicity in Jurkat cells. DCLP had immune stimulating effects, as evidenced by the DCLP-mediated increases in the level of DNA synthesis, viability, and cytokine secretion in mouse lymphocytes. Moreover, oral administration of DCLP produced a significant reduction in blood glucose level in alloxan-induced diabetic mice. These findings suggest that DCLP has a potential utility in treating patients who require enhanced antioxidation, immune function and/or hypoglycemic activity.
\end{abstract}

Keywords: Antioxidation, Anti-hyperglycemic, Dendrobium chrysotoxum Lindl, Immune stimulation, Polysaccharide, Traditional Chinese medicine

"Present address: College of Chemistry \& Chemical Engineering, Shanghai Jiao Tong University, Shanghai, 200240, P.R. China

*To whom correspondence should be addressed.

Tel: 82-63-270-4049; Fax: 82-63-270-4049

E-mail: leejc88@chonbuk.ac.kr

\section{Introduction}

Traditional oriental medicines have long been used to treat human diseases, particularly those associated with elderly patients. There has been increasing interest in examining the specific biological activity of traditional medicines, and numerous investigations support their antioxidant, immune stimulatory, antitumor, and anti-inflammatory activities (Satoh et al., 1996; Yamaguchi et al., 2000; Rao et al., 2006). Recent studies of traditional medicines have been carried out to identify their active ingredients and to test their efficacy in treating various diseases. As reported previously, several components isolated from traditional medicines have been proposed as useful therapeutic agents (Gong et al., 2004; Kim et al., 2005; Lee et al., 2005). Polysaccharides are one of the major active compounds responsible for the pharmacological activities contained in traditional herbal medicines ( $\mathrm{Li}$ et al., 2001; Xie et al., 2004; Fang et al., 2005).

The traditional Chinese herbs, Dendrobium, have been used in the preparation of herbal medicines in many Oriental countries for a long time. Over 60 species of Dendrobium are found in China, and many studies have demonstrated their beneficial activities in antioxidant, immune stimulating and antitumor activities (Ye and Zhao, 2002). Of the Dendrobium medical plants, Dendrobium chrysotoxum Lindl (DCL) is the most commonly used herb in the Chinese medicine, Herba Dendrobii (stem of Dendrobium) (Li et al., 2005). Although polysaccharide is believed to play an important role in the medicinal effects of DCL, its role as an antioxidant and in anti-hyperglycemic and immune stimulation had not been examined. Therefore, this study isolated polysaccharide from the stem of DCL and examined its antioxidant property in an attempt to define the mechanism of its antioxidant action using cell-free and cell-mediated radical generating systems. This study also determined whether the DCL polysaccharide (DCLP) has immune stimulating activity. Finally, the effects of DCLP on blood glucose level were evaluated using normal and alloxan-induced diabetic mice. 


\section{Materials and Methods}

Chemicals, plastics, and mice. Unless otherwise specified, all chemicals used in this study were purchased from Sigma Chemical Co. and all the plastics were obtained from Falcon Labware (Becton-Dickinson). Male BALB/c mice (6 weeks old) were obtained from Orient Co. Mice were housed in automatically controlled conditions with a 12-h light/dark cycle, $22 \pm 1^{\circ} \mathrm{C}$, and $45-55 \%$ relative humidity. All mice had free access to standard rodent pellet food and water ad libitum, only except when fasted before experiments. Experiments were carried out according to the guidelines of the Animal Care Committees of Chonbuk National University.

Isolation of polysaccharide. The dried sample of DCL was obtained from Shanghai Jiao Tong University after identification by botanists of this university. A voucher specimen (No. 2005-DCL) was also deposited at the herbarium of this university. Briefly, powdered DCL $(100 \mathrm{~g})$ was resuspended in absolute ethanol at $78^{\circ} \mathrm{C}$ for $1 \mathrm{~h}$ and then filtered through a filter paper (Whatman No. 3 ). The remainder was extracted three times with distilled water at $80^{\circ} \mathrm{C}$. The collected water extract was evaporated and reduced to a $200 \mathrm{ml}$ volume and then mixed with absolute ethanol $(1: 4, \mathrm{v} / \mathrm{v})$ for $24 \mathrm{~h}$ to precipitate the polysaccharide compounds. After centrifugation, the precipitates were collected and washed with absolute ethanol and acetone, respectively, and then lyophilized to $16 \mathrm{~g}$ (16\% of the initial amounts) to produce the dried polysaccharide sample of DCL (DCLP). DCLP was stored at $-20^{\circ} \mathrm{C}$ and freshly dissolved in distilled water immediately before use.

SDS-PAGE and FT-IR analysis. SDS-PAGE was performed using a Mini-Protean II cell (Bio-Rad) with $10 \%$ polyacrylamide gels. DCLP was dissolved in sample buffer in the absence of 2mercaptoethanol and analyzed without pre-boiling. The gels were stained with either with Coomassie brilliant blue (CBB) to detect proteins or with Schiff reagent to detect carbohydrates, as described elsewhere (Neville Jr and Glossmann, 1974). In addition, the molecular structure of DCLP was roughly determined by FT-IR (PerkinElmer, GX model).

Antioxidant assays using cell-free radical generating systems. A deoxyribose assay to determine the rate constant for the reactions between either antioxidants and hydroxyl radicals $\left({ }^{\circ} \mathrm{OH}\right)$ (referred to a non-site-specific scavenging assay) or antioxidants and iron ions (referred as a site-specific scavenging assay) was conducted as described by Halliwell et al. (1987). In addition, the ability of DCLP to reduce the $\mathrm{Fe}^{3+}$-EDTA complex to $\mathrm{Fe}^{2+}$-EDTA complex, acting as pro-oxidant, was examined using the non-site-specific scavenging assay mixture without the addition of ascorbic acid (Kim et al., 2005). DNA nicking assay was also performed using supercoiled pBR322 plasmid DNA prepared from DH5 $\alpha$ using Wizard ${ }^{\circledR}$ Plus SV Minipreps (Promega, Madison, WI), as described elsewhere (Lee et al., 2001). Finally, the scavenging activity on superoxide radical $\left(\mathrm{O}_{2}{ }^{-}\right)$was examined using a slight modification of the method described by Gotoh and Niki (1992).

Cell culture and treatment. Mouse splenocytes and Jurkat cells, or MCF-7 cells were cultured in RPMI-1640 or DMEM supplemented with $10 \%$ fetal bovine serum (FBS; HyClone, Logan, UT). When these cells had reached confluence, $1 \times 10^{5}-10^{6}$ cells per $\mathrm{ml}$ were resuspended in $100 \mu \mathrm{l}$ media and spread onto 96-well flat-bottomed plates. Before the DCLP treatment, the cultures were switched to a new medium supplemented only with $0.5 \%$ FBS. The cells were then processed for an analysis of the antioxidant activity, DNA synthesis, cytotoxicity, and cytokine production.

Cellular assay for antioxidant activity and DNA synthesis. Jurkat and glucose/glucose oxidase (G/GO) were used as the model cell and radical generating systems, respectively. Briefly, Jurkat cells $\left(1 \times 10^{5}\right.$ cells/well $)$ were placed onto 96 -well plates and exposed to the G/GO system $(10 \mathrm{mM}$ D-glucose and $10 \mathrm{mU} / \mathrm{ml}$ glucose oxidase in RPMI 1640 medium with $0.5 \%$ FBS) with or without DCLP. At various time points, cell viability was measured using a MTT assay system as described previously (Lee et al., 2006).

The level of DNA synthesis in the splenocytes, Jurkat, and MCF7 cells incubated with different DCLP concentrations was measured by adding $1 \mu \mathrm{Ci}$ of [methyl $\left.{ }^{3} \mathrm{H}\right]$ Thymidine deoxyribose (TdR; Amersham Pharmacia Biotech) to each well for 12-16 h during culture periods. Cells were collected with a cell harvester (Inotech Inc.), and the TdR content was determined using a liquid scintillation counter (Packard Instrument Co.).

Measurement of cytokine levels. The concentration of cytokines produced by lymphocytes after DCLP treatment was determined by ELISA. Briefly, the splenocytes cultured in 96-well tissue culture plates were exposed to different concentrations of DCLP for $72 \mathrm{~h}$. The culture supernatants were then collected and the level of cytokines representing the stimulation of Th1-type (IFN- $\gamma$ ) and Th2-type (IL-4 and IL-6) T cells, and macrophages (and/or B cells) (TNF- $\alpha$ ) was determined.

Characterization of DCLP. The DCLP was treated with pronase E to degrade the protein, or with $\mathrm{NaIO}_{4}$ to degrade the carbohydrate residues, as described elsewhere (Kim et al., 2002). A DCLP sample $(1 \mathrm{mg})$ was also incubated for $3 \mathrm{~h}$ at $37^{\circ} \mathrm{C}$ in $1 \mathrm{ml}$ of the culture medium containing 1,000 units of polymyxin $\mathrm{B}(\mathrm{PMB})$ to determine if DCLP contained lipopolysaccharide (LPS)-like compounds. In addition, $10 \mathrm{mg}$ of DCLP was dissolved in $1 \mathrm{ml}$ distilled water and the tube was soaked in a boiling water bath for $20 \mathrm{~min}$. The ability of the treated DCLP samples to stimulate lymphocyte proliferation was measured by a TdR uptake assay using mouse splenocytes.

Measurement of hypoglycemic activity in normal mice. Following an overnight fast, healthy mice (10 week-old) were divided into four groups $(n=7)$. All mice were then administrated orally and daily for one week with $0.5 \mathrm{ml}$ of phosphate-buffered saline (PBS) supplemented without (negative control) and with tolbutamide (80 $\mathrm{mg} / \mathrm{kg}$ body wt.) or DCLP (200 and $500 \mathrm{mg} / \mathrm{kg}$ body wt., respectively). Blood samples were drawn from the tail vein of the mice before the first administration and $1 \mathrm{~h}$ after the last administration of the samples. Blood glucose levels were determined using a commercially available quantification kit, AM201-K (Asan Pharmaceutical Co.). 
Diabetes induction and DCLP treatment. In order to evaluate the effects of DCLP on the blood glucose levels in diabetic mice, the overnight fasted 10-week-old BALB/c mice were made diabetic with alloxan. Briefly, alloxan monohydrate was dissolved in cold distilled water just before intraperitoneal injection, and the mice received alloxan at a dose of $160 \mathrm{mg} / \mathrm{kg}$ body weight. Diabetes was confirmed by measuring the blood glucose level after $16 \mathrm{~h}$ of fasting at 7 days post injection, and the mice with blood glucose concentration above $300 \mathrm{mg} / \mathrm{dl}$ were used in the experiments. The diabetic mice were divided into four groups ( 7 per group) and were orally administrated with tolbutamide or DCLP everyday for one week, as described above.

Statistical analyses. All the data is expressed as a mean \pm standard deviation (S.D.). A one-way ANOVA using SPSS ver. 10.0 software was used for multiple comparisons. A value of $p<0.05$ was considered significant.

\section{Results}

Properties of DCLP. The nature of DCLP was determined by separating the sample by SDS-PAGE and staining the gels with either $\mathrm{CBB}$ or Schiff reagent. The band with a molecular weight of approximately $150 \mathrm{kDa}$ was observed with Schiff reagent (Fig. 1A), while there was no a band stained with CBB (data not shown). This indicates that DCLP was not contaminated with any protein residues. Fig. 1B shows the FT-IR profile of DCLP. As shown in the figure, DCLP contained some special function groups, which means DCLP is a polysaccharide. For example, the peak at $3421 \mathrm{~cm}^{-1}$ was assigned to the $\mathrm{O}-\mathrm{H}$ stretching vibration, the two peaks at $2932 \mathrm{~cm}^{-11}$ and $1380 \mathrm{~cm}^{-1}$ were the $\mathrm{C}-\mathrm{H}$ stretching vibration, the peaks at $1737 \mathrm{~cm}^{-1}$ and $1250 \mathrm{~cm}^{-1}$ were the $\mathrm{C}=\mathrm{O}$ and $\mathrm{C}-\mathrm{O}$ stretching vibration, the peak at $1032 \mathrm{~cm}^{-1}$ was the $\mathrm{C}-\mathrm{O}$ of the C-O-C stretching vibration, and the peaks at $875 \mathrm{~cm}^{-1}$ and $813 \mathrm{~cm}^{-1}$ were Mannose.

Scavenging effect of DCLP on hydroxyl radicals and superoxide anions. The scavenging effect of DCLP on the hydroxyl radicals generated by $\mathrm{Fe}^{3+}$ ions was measured by determining the extent of deoxyribose degradation, which is an indicator of the formation of thiobarbituric acid-malonaldehyde (TBA-MDA) adduct (Fig. 2A). The concentration-dependent inhibition of hydroxyl radical-induced deoxyribose degradation was observed in both the site-specific and non-site specific assays (Fig. 2A, top and middle panels). However, more antioxidant activity was observed in the site-specific assay than in the non-site-specific assay when the same concentration of DCLP was used. The ability of DCLP to reduce the $\mathrm{Fe}^{3+}$-EDTA complex was also examined by measuring the level of deoxyribose degradation using the same reaction buffer without the addition of ascorbic acid. There was no increase in the formation of TBA-MDA by adding DCLP, while the addition of DCLP to the reaction buffer inhibited the TBA-MDA adduct in a dose-dependent manner (Fig. 2A, bottom panel).
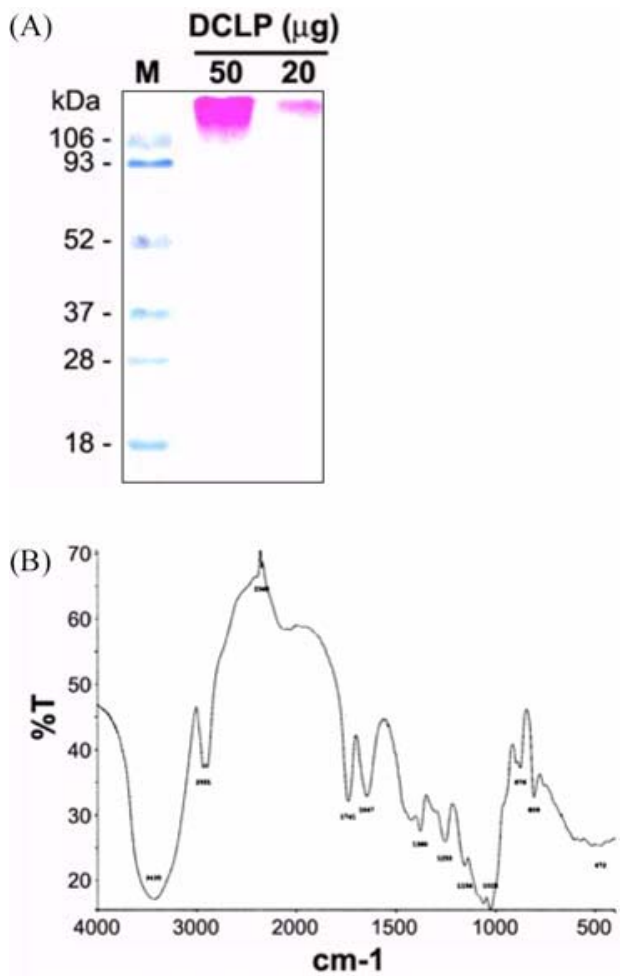

Fig. 1. SDS-PAGE and FT-IR analyses of Dendrobium chrysotoxum Lindl polysaccharides (DCLP). (A) DCLP was loaded at the indicated doses, electrophoresed, and stained with Schiff reagent. $M$ represents a pre-stained molecular weight marker. (B) In addition, the DCLP was analyzed by FT-IR.

To further measure the scavenging effect of DCLP on $\mathrm{Fe}^{3+}$ dependent hydroxyl radicals, this study investigated whether or not the sample reduced $\mathrm{Fe}^{3+}$-dependent DNA nicking (Fig. 2B). When pBR322 plasmid DNA was dissolved in the reaction mixture, a time-dependent increase in the formation of single-stranded nicked DNA (Form II) was observed (data not shown). However, the addition of DCLP to the nicking reaction mixture inhibited Form II DNA formation thereby increasing the level of Form I DNA.

The scavenging effect of DCLP on superoxide anions was determined by monitoring the reduction of NBT induced by superoxide anions produced by the xanthine oxidase-mediated degradation of hypoxanthine. DCLP inhibited NBT reduction in a dose-dependent manner (Fig. 2C). The addition of $4 \mathrm{mg} /$ $\mathrm{ml}$ DCLP to the reaction solution inhibited the production of superoxide anions by $50.5 \%$. DCLP alone did not alter the absorbance of the reaction solution containing only NBT, suggesting that DCLP did not directly reduce NBT (data not shown). In addition, DCLP significantly inhibited the activity of DPPH radicals in a dose-dependent manner (data not shown).

Protective effect of DCLP on cytotoxicity induced by glucose oxidase-mediated oxidative stress. The antioxidant activity of DCLP was further examined using the G/GO 
(A)
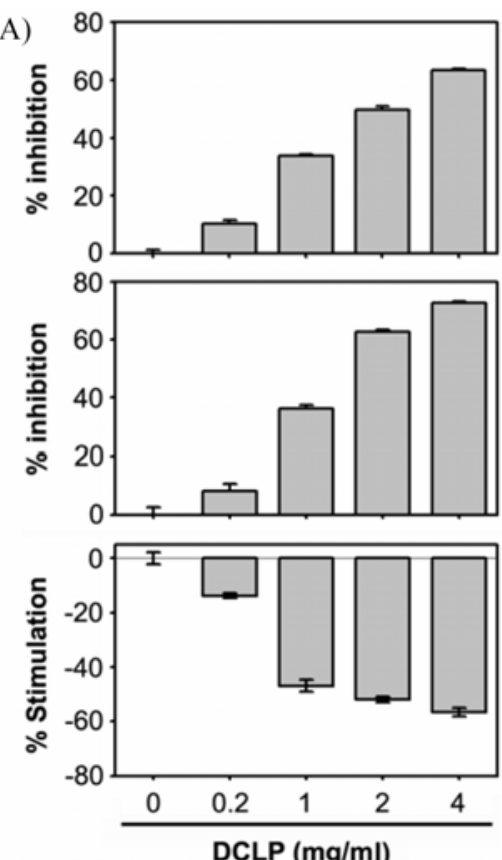

(B)

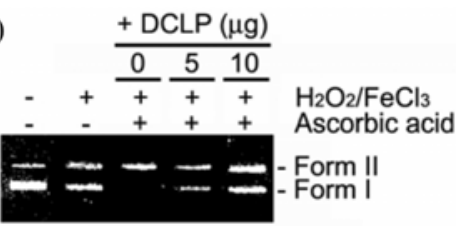

(C)

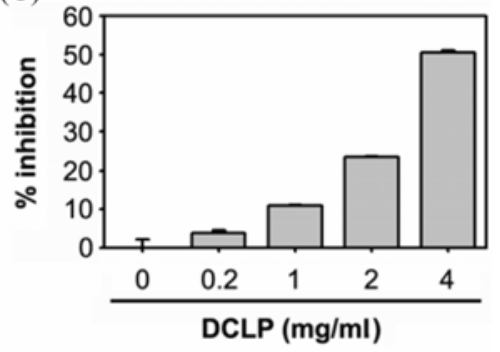

Fig. 2. Antioxidant potential of DCLP on cell-free radical generating systems. (A) Inhibitory effects of DCLP on deoxyribose degradation induced by hydroxyl radicals. The direct scavenging activity of hydroxyl radicals (top panel) and the chelating activity of iron ions by DCLP (middle panel) are expressed as the \% inhibition. The ability of DCLP to reduce the Fe ${ }^{3+}$-EDTA complex to Fe ${ }^{2+}$ EDTA complex was also tested (bottom panel), and the data is expressed as the \% stimulation. (B) Inhibitory effect of DCLP on DNA nicking caused by hydroxyl radicals. (C) Inhibitory effect of DCLP on NBT reduction induced by superoxide anions.

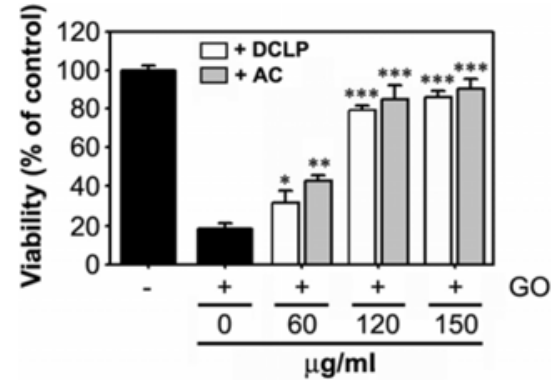

Fig. 3. Protective effects of DCLP on glucose oxidase (GO)mediated oxidative stress. Jurkat cells were exposed to the $\mathrm{G} / \mathrm{GO}$ system for $24 \mathrm{~h}$ in the presence of DCLP or acteoside, and cell viability was analyzed by the MTT assay. Acteoside was used as the control antioxidant. ${ }^{*} p<0.05, * * p<0.01$, and $* * * p<0.001$ vs. GO treatment alone. AC, acteoside.

system, in which GO yields hydrogen peroxide via a catalytic reaction from its substrate, glucose (Fig. 3). DCLP significantly protected the Jurkat cells from G/GO-mediated cytotoxicity, and the protective activity was quite similar to that of acteoside, which used as the control antioxidant because its antioxidant potential was much higher than that of ascorbic acid (Kim et al., 2005). The addition of 60 and $120 \mu \mathrm{g} / \mathrm{ml}$ DCLP increased the viability by $30.9 \% \pm 5.2(p<0.05)$ and $78.9 \% \pm 2.7(p<0.001)$, respectively, compared with that of the G/GO treatment alone $(18.3 \%)$.
Stimulating effects of DCLP on proliferation, viability, and cytokine secretion of mouse lymphocytes. The effect of DCLP on DNA synthesis in various types of cells was determined using a tritium incorporation assay. DCLP significantly increased the level of DNA synthesis in the splenocytes in that a treatment with $50 \mu \mathrm{g} / \mathrm{ml}$ DCLP increased the TdR uptake 6.72-fold $(p<0.001)$ compared with the untreated control $(1,329 \mathrm{cpm})$ (Fig. 4A). However, this increase was not observed in the Jurkat cells and MCF-7 cells.

In order to determine if DCLP itself has a cytotoxic effect on lymphocytes, the splenocytes were treated with various DCLP concentrations and incubation times. When the cells were exposed to DCLP for $36 \mathrm{~h}$, the level of MTT reduction increased significantly $(p<0.05)$ in all the treatments compared with the untreated control cells (Fig. 4B, upper panel). In addition, the MTT reducing activity of splenocytes was not decreased even when treated with $200 \mu \mathrm{g} / \mathrm{ml}$ DCLP for $48 \mathrm{~h}$ (Fig. 4B, below panel).

The splenocyte-specific stimulating activity of DCLP was further examined by measuring the level of cytokines produced by DCLP in the cells (Fig. 4C). A prominent increase in IFN$\gamma$ and IL- 6 production was observed by treating them with DCLP. When the cells were exposed to $40 \mu \mathrm{g} / \mathrm{ml}$ DCLP for $72 \mathrm{~h}, 11.7 \mathrm{ng} / \mathrm{ml} \mathrm{IFN}-\gamma$ and $34.2 \mathrm{ng} / \mathrm{ml} \mathrm{IL-6}$ were produced

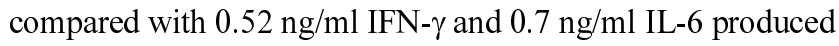
in the untreated control cells, respectively. The DCLP treatment also increased the production of TNF- $\alpha$ compared 
(A)

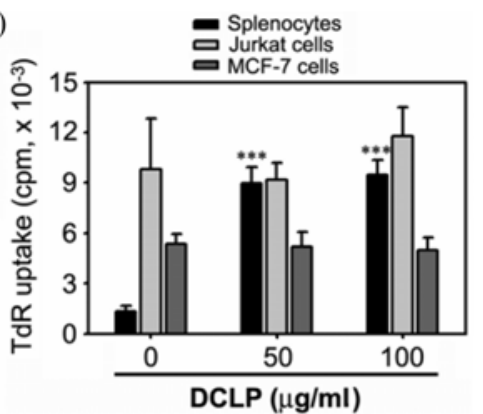

(B)

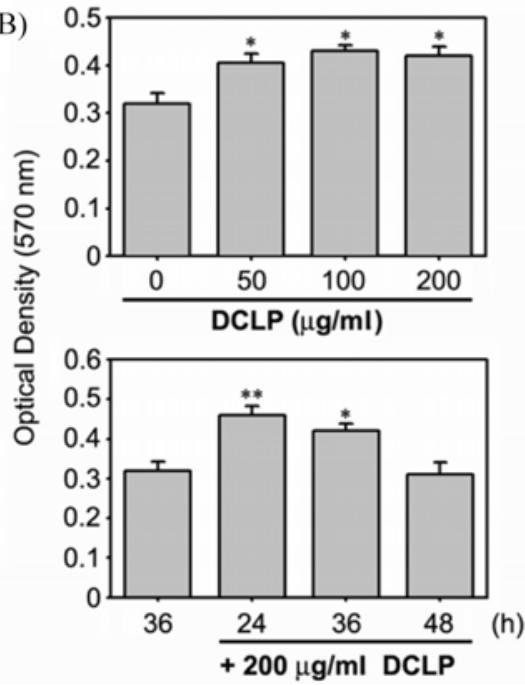

(C)
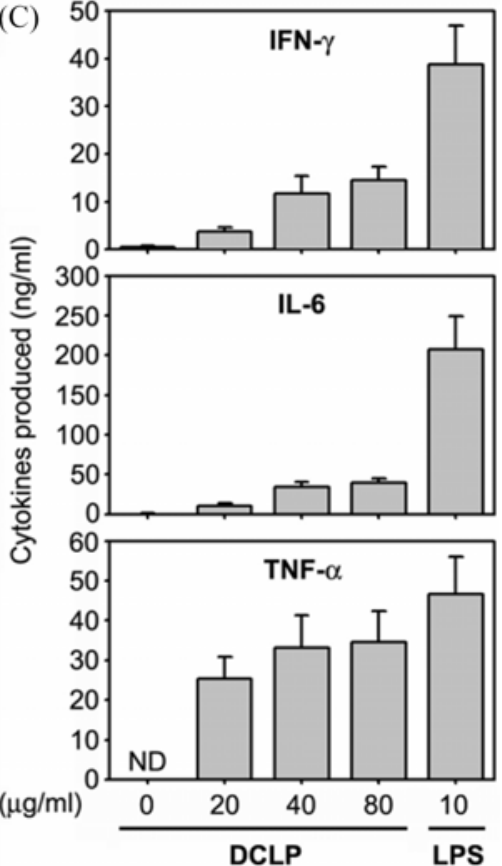

Fig. 4. Immune stimulating effects of DCLP. (A) Effects of DCLP on cell proliferation in various types of cells. The cells were incubated with the indicated concentrations of DCLP and exposed to $1 \mu \mathrm{Ci} / \mathrm{ml}\left[\right.$ methyl $\left.{ }^{3} \mathrm{H}\right] \mathrm{TdR}$ for the last $12 \mathrm{~h}$ of the $48 \mathrm{~h}$ culture period. $* * * p<0.001$ vs. control. (B) Effects of DCLP on MTT-reducing activity of splenocytes. Splenocytes were incubated with the indicated concentrations of DCLP for $36 \mathrm{~h}$ (upper panel) or the indicated times with $200 \mathrm{mg} / \mathrm{ml}$ DCLP (below panel) and processed for MTT staining. ${ }^{*} p<0.05$ and $* * p<0.01$ vs. control. (C) Effects of DCLP on cytokine production by mouse splenocytes. The cells were exposed to the indicated amounts of DCLP or lipopolysaccharide (LPS) for $72 \mathrm{~h}$ and the levels of IFN- $\gamma$ (top panel), IL-6 (middle panel), and TNF- $\alpha$ (bottom panel) were analyzed by ELISA. LPS was used as the positive control. ND, not detected.

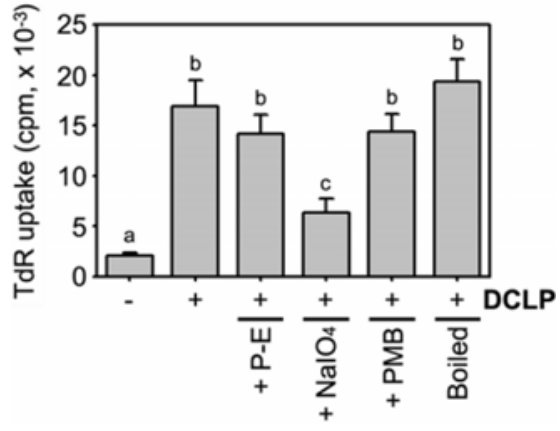

Fig. 5. Effects of pronase $\mathrm{E}, \mathrm{NaIO}_{4}$, polymyxin $\mathrm{B}$, and heating on the splenocyte-stimulating activity of DCLP. The ability of the treated DCLP samples to stimulate lymphocyte proliferation was measured by a TdR uptake assay. Here, $50 \mathrm{mg} / \mathrm{ml}$ of the DCLP samples was added to the splenocytes cultures. The superscript letters indicate the significant differences $(p<0.05)$ between the experimental and control values. P-E, pronase E; $\mathrm{PMB}$, polymyxin $\mathrm{B}$. with the control, such that the level of TNF- $\alpha$ production was similar to that obtained after treating the splenocytes with LPS $(46.7 \mathrm{ng} / \mathrm{ml})$. However, no change in the level of IL-4 was detected after stimulating the splenocytes with DCLP (data not shown).

Characterization of DCLP on lymphocyte stimulation. In order to further clarify whether or not the splenocytestimulating activity of DCLP was due to its carbohydrate residue, the sample was treated with either pronase $\mathrm{E}, \mathrm{NaIO}_{4}$, or PMB, and their ability to stimulate the splenocytes was examined. As shown in Fig. 5, the treatment of DCLP with $\mathrm{NaIO}_{4}$ but not pronase $\mathrm{E}$ inhibited the stimulating activity of the sample. PMB, which is an inhibitor of the LPS-like mitogen-mediated response, did not reduce the DCLP activity. In contrast, the same PMB treatment significantly inhibited the LPS-mediated increase in $\mathrm{TdR}$ uptake by splenocytes (data not shown). Finally, DCLP was boiled for $20 \mathrm{~min}$ and its ability to stimulate lymphocyte proliferation was measured. 


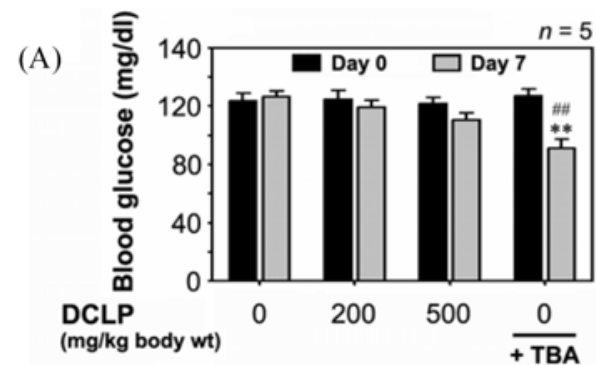

(B)

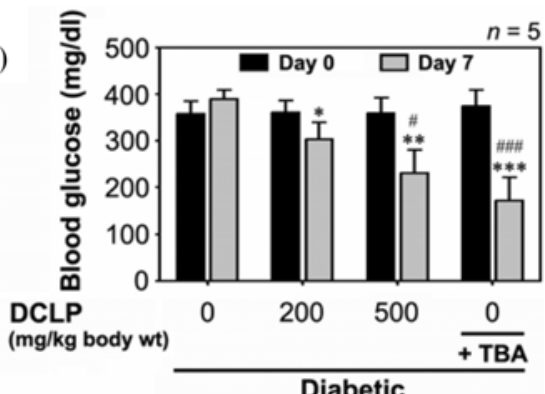

Fig. 6. Hypoglycemic effect of DCLP in normal and diabetic mice. Healthy normal (A) and alloxan-induced diabetic mice (B) were administrated with the DCLP or tolbutamide, and blood samples were collected from the mice before the first administration and $1 \mathrm{~h}$ after the last administration of the samples and were processed for the analysis of glucose concentrations, as described in the Materials and methods section. $* p<0.05$, ${ }^{* *} p<0.01$, and ${ }^{* * *} p<0.001$ vs. control. ${ }^{*} p<0.05,{ }^{\#} p<0.01$, and ${ }^{\# \#} p<0.001$ vs. day 0 . TBA, tolbutamide.

The splenocyte-stimulating activity of DCLP was not affected by boiling. However, concanavalin A activity was inhibited significantly by the treatment (data not shown).

\section{Anti-hyperglycemic effect of DCLP on blood glucose level} in normal and alloxan-induced diabetic mice. It has been shown that polysaccharides from traditional herbal medicine have an anti-hyperglycemic activity in streptozotocin- or alloxan-induced diabetic animals (Xie et al., 2004; Li et al., 2006). Therefore, this study was also designed to determine whether DCLP have an anti-diabetic activity in alloxaninduced hyperglycemic mice. As shown in Fig. 6A, there was no significant difference between the basal blood glucose level in both the control and experimental groups. On day 7, however, tolbutamide-treated mice had significantly lower blood glucose concentrations $(91.2 \pm 13.6 \mathrm{mg} / \mathrm{dl} ; p<0.01)$, as compared to the PBS-treated control mice $(126.4 \pm 8.5 \mathrm{mg} /$ dl).

Alloxan-treated mice had dramatically high basal blood glucose level (approximately $360 \mathrm{mg} / \mathrm{dl}$ ). In contrast, DCLP treatment decreased significantly blood glucose levels such that seven days administration of DCLP at doses of 200 and $500 \mathrm{mg} / \mathrm{kg}$ body weight reduced the levels up to $303.6 \pm 35.8$ $(p<0.05)$ and $231.7 \pm 49.4 \mathrm{mg} / \mathrm{dl}(p<0.01)$, respectively, as compared to the alloxan treatment alone $(389.2 \pm 19.4 \mathrm{mg} / \mathrm{dl})$
(Fig. 6B). Tolbutamide also had a significant anti-hyperglycemic activity $(p<0.001)$. However, body weight of the mice was not changed by treating DCLP itself during the experimental periods (data not shown).

\section{Discussion}

For a long time, plant-derived antioxidants have been used to reduce the level of oxidative stress within the human body (Rice-Evans et al., 1995; van Poppel and van den Berg, 1997). Although macromolecules such as proteins and polysaccharides have been shown to be the major components associated with the immune stimulating activity of traditional herbal plants, recent studies have also demonstrated their potential, particularly their antioxidant (Ghosh et al., 2006). The polysaccharide isolated from cultured Cordyceps, which is one of the most valued traditional Chinese medicines, has strong antioxidant activity against superoxide radicals and protects hydrogen peroxide-induced cytotoxicity of PC12 cells (Li et al., 2003). In addition, the polysaccharide present in traditional Chinese medicine Ganoderma lucidum, which is used for the immune dysfunction related to illness, reduces the formation of DPPH radicals (Xie et al., 2006). This means that polysaccharide also has a potential antioxidant role.

Hydroxyl radicals are the most reactive of all the reduced forms of dioxygen and are believed to initiate cell damage both in vitro and in vivo (Rollet-Labelle et al., 1998). Thus, we initially used the $\mathrm{Fe}^{3+}$-dependent system to examine the ability of DCLP to scavenge hydroxyl radicals generated by iron. In the system, ascorbic acid acts as an electron donor rather than an antioxidant on account of its high redox activity and thus facilitates the production of hydroxyl radicals via the reduction of $\mathrm{Fe}^{3+}$-EDTA complex (Aruoma et al., 1997). DCLP inhibited deoxyribose degradation more by chelating iron ions than by scavenging hydroxyl radicals directly. The scavenging activity of DCLP was also confirmed using a DNA nicking assay, which indicates that DCLP effectively mitigates the oxidative stresses on susceptible biomolecules, such as DNA. In addition, the ability of DCLP to reduce the $\mathrm{Fe}^{3+}$-EDTA complex was not observed. This suggests that DCLP is a potential antioxidant but does not possess the redox potential to act as an electron donor that generates hydroxyl radicals in the deoxyribose assay (Laughton et al., 1989). Therefore, it is believed that the antioxidant property of DCLP is different from those of ascorbic acid and acteoside, which are powerful antioxidants with oxidoreductive potential (Kim et al., 2005).

Superoxide anions are the most common free radicals in vivo and are generated in a variety of biological systems by either auto-oxidation processes or by enzymes. The concentration of superoxide anions increases under oxidative stress and related situations (von Harsdorf et al., 1999; Matés et al., 2002). Moreover, superoxide anions produce other types of cell-damaging free radicals and oxidizing agents, and 
indirectly initiate lipid peroxidation (Yen and Heieh, 1997; Liu and Ng, 2000). In the NBT system, xanthine oxidase, which is one of the main enzymatic sources of ROS in vivo, generates superoxide radicals, which then reduces NBT to yield blue formazan (Gotoh and Niki, 1992). The NBT reduction was actively inhibited by DCLP, which suggests that the DCLP is a potential scavenger of superoxide anions and hydroxyl radicals.

In order to further evaluate the antioxidant property of DCLP in living cells, the degree to which DCLP protects against radical-induced cell injury was tested in Jurkat cells using a MTT assay system. DCLP significantly prevented the hydroxyl radical-mediated cell death. Considering the fact that hydrogen peroxide induces apoptosis through the generation of hydroxyl radicals, it is suggested that DCLP can inhibit ROS-mediated apoptosis in cells. It is also believed that DCLP inhibits the GO-mediated cell death by chelating intracellular metal ions rather than by scavenging superoxide anions directly. This is because more hydroxyl radicals are generated through the Fenton reaction in the G/GO system than through the Haber-Weiss reaction (Lee et al., 2006).

Several active compounds regulate the induction of immune response. In particular, polysaccharides can act as an immune stimulator (Fang et al., 1985). However, there are reports showing that polysaccharides have dual functions to act as both an inhibitor and stimulator of proliferation ( $\mathrm{Li}$ et al., 2006; Xie et al., 2006). This contrary action of polysaccharide is believed to be derived from their structures and chemical properties, and types of cells studied. In this study, DCLP activated the proliferation of splenocytes. This was found to be a cell-specific response, as evidenced by the lack of proliferation observed in Jurkat and MCF-7 cells. In addition, treating the splenocytes with DCLP increased the level of IFN- $\gamma$, IL- 6 and TNF- $\alpha$, thereby indicating that DCLP induces the direct stimulation of $\mathrm{B}$ lymphocytes and/or macrophages as well as T lymphocytes. Moreover, this study showed that the DCLP did not contain LPS-like compounds or lectin compounds, and the stimulating activity of the sample was attributed to the carbohydrate residues contained in DCLP. This finding is in good agreement with the results from a previous study (Kim et al., 2002).

Diabetes is one of the major health problems in the population worldwide. Many studies have been focused on the development of a new anti-diabetic agent from dietary and medicinal plants. In particular, polysaccharides were found to have a significant anti-hyperglycemic activity (Kiho et al., 1999; Xie et al., 2004). This study also showed that DCLP treatment significantly reduced the levels of blood glucose in alloxan-induced diabetic mice. Alloxan induces type I diabetes mellitus by disrupting the balance between cellular antioxidant defenses and free radical formation as well as by partially destructing $\beta$-cells in the pancreas (Abdel-Barry et al., 1997; Szkudelski et al., 1998). This postulates that polysaccharides having high antioxidant potential may have a role to prevent the development of diabetes (Li et al., 2006).
Therefore, this study suggests that DCLP may play a beneficial role in treating diabetic patients, and its role is closely related to the antioxidant potential. However, the precise mechanism by which DCLP reduces blood glucose levels in diabetic mice will require further detailed study.

In conclusion, oxidative stress may play a role in aging as well as in several degenerative diseases. The finding that DCLP with a molecular mass of approximately $150 \mathrm{kDa}$ has potent antioxidant, immune stimulating, and anti-hyperglycemic properties highlights the potential use of DCLP as a therapeutic agent for diabetic patients. Studies aimed at identifying the physicochemical property of DCLP, further elucidating the intracellular signaling mechanism of DCLP and determining its pharmacological abilities in vivo are currently underway.

Acknowledgment This work was supported by the Korea Research Foundation Grant endowed to the RCBM of Chonbuk National University (KRF2006-005-J03102).

\section{References}

Abdel-Barry, J. A., Abdel-Hassan, I. A. and Al-Hajiem, M. H. H. (1997) Hypoglycemic and antihyperglycemic effects of Trigonella foenum-graecum leaf in normal and alloxan induced diabetic rats. J. Ethnopharmacol. 58, 149-155.

Aruoma, O. I., Spencer, J. P. E., Warren, D., Jenner, P., Butler, J. and Halliwell, B. (1997) Characterization of food antioxidants, illustrated using commercial garlic and ginger preparations. Food Chem. 60, 149-156.

Fang, J. N., Proksch, A. and Wagner, H. (1985) Immunologically active polysaccharides of Acanthopanax senticosus. Phytochemistry 24, 2619-2622.

Fang, X., Yu, M. M., Yuen, W. H., Zee, S. Y. and Chang, R. C. (2005) Immune modulatory effects of Prunella vulgaris L. on monocytes/macrophages. Int. J. Mol. Med. 16, 1109-1116.

Ghosh, A., Sarkar, K. and Sil, P. C. (2006) Protective effect of a $43 \mathrm{kD}$ protein from the leaves of the herb, Cajanus indicus $\mathrm{L}$ on chloroform induced hepatic-disorder. J. Biochem. Mol. Biol. 39, 197-207.

Gong, Y. Q., Fan, Y., Wu, D. Z., Yang, H., Hu, Z. B. and Wang, Z. T. (2004) In vivo and in vitro evaluation of erianin, a novel anti-angiogenic agent. Eur. J. Cancer 40, 1554-1565.

Gotoh, N. and Niki, E. (1992) Rates of interactions of superoxide with vitamin $\mathrm{E}$, vitamin $\mathrm{C}$ and related compounds as measured by chemiluminescence. Biochim. Biophys. Acta 1115, 201-207.

Halliwell, B., Gutteridge, J. M. C. and Aruoma, O. I. (1987) The deoxyribose method: simple "test-tube" assay for determination of rate constants for reactions of hydroxyl radicals. Anal. Biochem. 165, 215-219.

Kiho, T., Ookubo, K., Usui, S., Ukai, S. and Hirano, K. (1999) Structural features and hypoglycemic activity of a polysaccharide (CS-F10) from the cultured mycelium of Cordyceps sinensis. Biol. Pharm. Bull. 22, 966-970.

Kim, E. H., Lee, J. C., Park, C. H., Lee, M. Y. and Jang, Y. S. (2002) Soamsan, a traditional Korean medicine, enhances antigen-specific immune responses in low-responder mice via 
the combined activity of glycoproteins and endotoxins. Int. Immunopharmacol. 2, 1529-1540.

Kim, S. S., Son, Y. O., Chun, J. C., Kim, S. E., Chung, G. H., Hwang, K. J. and Lee, J. C. (2005) Antioxidant property of an active component purified from the leaves of paraquat-tolerant Rehmannia glutinosa. Redox Report 10, 311-318.

Laughton, M. J., Halliwell, B., Evans, P. J. and Hoult, J. R. (1989) Antioxidant and pro-oxidant actions of the plant phenolics quercetin, gossypol and myricetin: Effects on lipid peroxidation, hydroxyl radical generation and bleomycin-dependent damage to DNA. Biochem. Pharmacol. 38, 2859-2865.

Lee, J. C., Kim, J., Lim, K. T., Yang, M. S. and Jang, Y. S. (2001) Ethanol eluted extract of Rhus verniciflua Stokes showed both antioxidant and cytotoxic effects on mouse thymocytes depending on the dose and time of the treatment. J. Biochem. Mol. Biol. 34, 250-258.

Lee, J. C., Lee, K. Y., Jung, H. N., Kim, J. G. and Jang, Y. S. (2005) Glycoproteins contained within Soamsan, a traditional oriental medicine, are the main class of active ingredients responsible for the medicine-induced immune stimulation. $J$. Biochem. Mol. Biol. 38, 253-257.

Lee, J. C., Son, Y. O., Choi, K. C. and Jang, Y. S. (2006) Hydrogen peroxide induces apoptosis of BJAB cells due to formation of hydroxyl radicals via intracellular iron-mediated Fenton chemistry in glucose oxidase-mediated oxidative stress. Mol. Cells 22, 21-29.

Li, S. P., Li, P., Dong, T. T. X. and Tsim, K. W. K. (2001) Antioxidation activity of different types of natural Cordyceps sinensis and cultured Cordyceps mycelia. Phytomedicine 8, 207-212.

Li, S. P., Zhang, G. H., Zeng, Q., Huang, Z. G., Wang, Y. T., Dong, T. T. X. and Tsim, K. W. K. (2006) Hypoglycemic activity of polysaccharide, with antioxidation, isolated from cultured Cordyceps mycelia. Phytomedicine 13, 428-433.

Li, S. P., Zhao, K. J., Ji, Z. N., Song, Z. H., Dong, T. T. X., Lo, C. K., Cheung, J. K. H., Zhu, S. Q. and Tsim, K. W. K. (2003) A polysaccharide isolated from Cordyceps sinensis, a traditional Chinese medicine, protects PC12 cells against hydrogen peroxide-induced injury. Life Sci. 73, 2503-2513.

Li, T., Wang, J. and Lu, Z. (2005) Accurate identification of closely related Dendrobium species with multiple speciesspecific gDNA probes. J. Biochem. Biophys. Methods 62, 111123.

Liu, F. and Ng, T. B. (2000) Antioxidative and free radical scavenging activities of selected medicinal herbs. Life Sci. 66, 725-735.

Matés, J. M., Pérez-Gómez, C., Núñez de Castro, I., Asenjo, M. and Márquez, J. (2002) Glutamine and its relationship with intracellular redox status, oxidative stress and cell proliferation/ death. IJBCB 34, 439-458.

Neville Jr, D. M. and Glossmann, H. (1974) Molecular weight determination of membrane protein and glycoprotein subunits by discontinuous gel electrophoresis in dodecylsulfate. Method. Enzymol. 32, 92-102.

Rao, Y. K., Fang, S. H. and Tzeng, Y. M. (2006) Anti-inflammatory activities of constituents isolated from Phyllanthus polyphyllus. J. Ethnopharmacol. 103, 181-186.

Rice-Evans, C. A., Miller, N. J., Bolwell, P. G., Bramley, P. M. and Pridham, J. B. (1995) The relative antioxidant activities of plant-derived polyphenolic flavonoids. Free Radic. Res. 22, 375-383.

Rollet-Labelle, E., Grange, M. J., Elbim, C., Marquetty, C., Gougerot-Pocidalo, M. A. and Pasquier, C. (1998) Hydroxyl radical as a potential intracellular mediator of polymorphonuclear neutrophil apoptosis. Free Radic. Biol. Med. 24, 563-572.

Satoh, K., Nagai, F., Ushiyama, K. and Kano, I. (1996) Specific inhibition of $\mathrm{Na}+, \mathrm{K}(+)$-ATPase activity by atractylon, a major component of Byaku-jutsu, by interaction with enzyme in the E2 state. Biochem. Pharmacol. 51, 339-343.

Szkudelski, T., Kandulska, K. and Okulicz, M. (1998) Alloxan in vivo does not only exert deleterious effects on pancreatic B cells. Physiol. Res. 47, 343-346.

van Poppel, G. and van den Berg, H. (1997) Vitamins and cancer. Cancer Lett. 114, 195-202.

von Harsdorf, R., Li, P. F. and Dietz, R. (1999) Signaling pathway in reactive oxygen species-induced cardiomyocyte apoptosis. Circulation 99, 2934-2941.

Yamaguchi, Y., Kagota, S., Nakamura, K., Shinozuka, K. and Kunitomo, M. (2000) Antioxidant activity of the extracts from fruiting bodies of cultured Cordyceps sinensis. Phytother. Res. 14, 647-649.

Yen, G. C. and Heieh, C. L. (1997) Antioxidant effects of dopamine and related compounds. Biosci. Biotechnol. Biochem. 61, 1646-1649.

Ye, Q. H. and Zhao, W. M. (2002) New alloaromadendrane, cadinene and cyclocopacamphane type sesquiterpene derivatives and bibenzyle from Dendrobium nobile. Planta Med. 68, 723729.

Xie, J. T., Wu, J. A., Mehendale, S., Aung, H. H. and Yuan, C. S. (2004) Anti-hyperglycemic effect of the polysaccharide fraction from American ginseng berry extract in $o b / o b$ mice. Phytomedicine 11, 182-187.

Xie, J. T., Wang, C. Z., Wicks, S., Yin, J. J., Kong, J., Li, J., Li, Y. C. and Yuan, C. S. (2006) Ganoderma lucidum extract inhibits proliferation of SW 480 human colorectal cancer cells. Exp. Oncol. 28, 25-29. 